\title{
Continuous Geodesic Convolutions for Learning on 3D Shapes
}

\author{
Zhangsihao Yang ${ }^{1}$, Or Litany ${ }^{1}$, Tolga Birdal ${ }^{1}$, Srinath Sridhar $^{1}$, Leonidas Guibas ${ }^{1, *}$ \\ ${ }^{a}$ Stanford University
}

\begin{abstract}
The majority of descriptor-based methods for geometric processing of non-rigid shape rely on handcrafted descriptors. Recently, learning-based techniques have been shown effective, achieving state-of-the-art results in a variety of tasks. Yet, even though these methods can in principle work directly on raw data, most methods still rely on hand-crafted descriptors at the input layer. In this work, we wish to challenge this practice and use a neural network to learn descriptors directly from the raw mesh. To this end, we introduce two modules into our neural architecture. The first is a local reference frame (LRF) used to explicitly make the features invariant to rigid transformations. The second is continuous convolution kernels that provide robustness to sampling. We show the efficacy of our proposed network in learning on raw meshes using two cornerstone tasks: shape matching, and human body parts segmentation. Our results show superior results over baseline methods that use hand-crafted descriptors.
\end{abstract}

Keywords: Geometric Deep Learning, Shape Descriptors, Shape Segmentation, Shape Matching

\section{Introduction}

Shape descriptors are key to many applications in 3D computer vision and graphics. Examples include shape matching, segmentation, retrieval, and registration, to name a few. A good local descriptor should balance between two opposite forces. On the one hand, it needs to be discriminative enough to uniquely describe a surface local region. At the same time though, it needs to keep robustness to nuisance factors like noise or sampling. Depending on the task, other properties may be required. Common examples are invariance to rigid transformations (Deng et al., 2019, 2018a,b) or isometric deformations (Masci et al., 2015; Boscaini et al., 2016a; Monti et al., 2017). To this end, many descriptors have been manually crafted with built-in invariance. However, these rely on one's ability to analytically model structure in the data which can often be too difficult. Alternatively, machine learning approaches and neural networks in particular, can recover complex patterns from training samples. Further, end-to-end learning is task aware and thus can tailor the learned descriptors to the specified task. Neural networks have proven very powerful in learning descriptors from raw data in various domains including images, text, audio and point clouds. Recently, an exciting research branch termed geometric deep learning has emerged, offering various techniques to process shape represented as meshes. Interestingly though, the vast majority of methods still rely on hand-crafted descriptors at the input to the network as these seem to perform better than working on raw data. In this work, we wish to challenge this practice and learn directly from the raw mesh. Our proposed method is data-driven in nature, however, we integrate the powerful local reference frame (LRF) module commonly used in hand-crafted descriptors into our network. We found through experimentation that structuring the learning through the LRF, is key to reach good performance. A second contribution is the use of continuous convolution kernels. This concept was recently shown to be quite powerful in point cloud networks Wang

\footnotetext{
* Corresponding author

Email addresses: zhangsiy@cs.stanford.edu (Zhangsihao Yang), or.litany@gmail.com (Or Litany), tbirdal@stanford.edu (Tolga Birdal), ssrinath@cs.stanford.edu (Srinath Sridhar), guibas@cs.stanford.edu (Leonidas Guibas)
} 
et al. (2018). Here, we show its usefulness in the context of deformable meshes. We show the efficacy of our proposed network in learning on raw meshes using two cornerstone tasks: shape matching, and human body parts segmentation. Our results show superior results over baseline methods that use hand-crafted descriptors.

Contributions Our contributions can be summarized as follows.

- We introduce a local reference frame $(\mathrm{LRF})$ and continuous convolution kernel modules in the context of deformable shapes.

- Using these, we are able to work directly on raw mesh features and outperform previous methods that take hand-crafted descriptors as input.

- We achieve improved results on deformable shape matching, and human body part segmentation.

\section{Related Work}

\subsection{Shape descriptors}

Rigid Case Rotation invariant 3D local descriptors are of great interest in the realm of 3D computer vision. Most of the works consider the scenario where the 3D point sets undergo a rigid transformation. The first handcrafted family of works tried to achieve repeatability under those transformations by building certain invariances such as isometry invariance (Tombari et al., 2010b; Johnson and Hebert, 1999; Guo et al., 2013; Rusu et al., 2008, 2009; Tombari et al., 2010a). Many of these works rely extensively on a local reference frame that is assumed to be repeatably constructed on the point sets (Petrelli and Di Stefano, 2011; Mian et al., 2010; Melzi et al., 2019). With the advances in deep networks, these methods have been replaced by their learned counterparts (Zeng et al., 2017; Khoury et al., 2017; Deng et al., 2018b,a; Gojcic et al., 2019; Choy et al., 2019). Be it data driven or not, a large portion of all these works owe their robustness to the local reference frames unless the input is made invariant to rotations (Deng et al., 2018a; Zhao et al., 2019). One of the aims of this paper is to extend the findings regarding locally rigid LRFs to the non-rigid.

Case of Deformable Shapes For the non-rigid shapes, pointwise descriptors are mostly designed to be intrinsic in order to handle isometric deformations (Aubry et al., 2011; Rustamov, 2007; Sun et al., 2009) and scale (Bronstein and Kokkinos, 2010). However, designing a descriptor by hand is a cumbersome task. It requires manual balancing of the trade-off between robustness and discriminability, and often relies on heuristics to capture local patterns. Learning based methods are well suited for this task, given the availability of enough training samples. Pioneering works in the field include Bronstein et al. (2011) which extended a "bag of features" approach to non-rigid 3D shape retrieval, and Litman and Bronstein (2013) which utilized a Mahalanobis metric learning to optimize a parametric spectral descriptor for shape matching. The success of deep learning in computer vision, has motivated a new active research area termed Geometric Deep Learning (Bronstein et al., 2017). A main challenge in this field is how to construct basic operations such as convolution and pooling for geometric data structures. One line of work has opted for extracting geodesic patches (Masci et al., 2015; Boscaini et al., 2016a; Monti et al., 2017). This way, the convolution operator is defined intrinsically on the manifold guaranteeing invariance to isometric deformations. The challenge lies in constructing repeatable local patches with a canonical orientation. For nonrigid shapes these are often based on curvature, but other approaches exist such as Huang et al. (2015) and the recently proposed GFrames (Melzi et al., 2019). On the other hand, spectral techniques Bruna et al. (2013); Henaff et al. (2015) generalize a convolutional network through the Graph Fourier transform, thus avoiding the need for a patch. A polynomial parameterization of the learned filters was proposed in Defferrard et al. (2016) in order to spatially localize the kernel and reduce the learning complexity. Common to most learning based methods, is that they use precomputed descriptors as inputs and improve upon them through learned operations. Few works explored directly using raw mesh data. In Poulenard and Ovsjanikov (2019) it was shown that using 3D coordinates underperform SHOT (Tombari et al., 2010b) as input; while in Verma et al. (2018) the opposite conclusion was reached. MeshCNN (Hanocka et al., 2019) defined convolution on triangular meshes treating the edge as a first citizen, rather than the nodes. Using angles and edge ratios as input features they were able to work on the raw mesh while being rotation, translation and (uniform) scale invariant. 


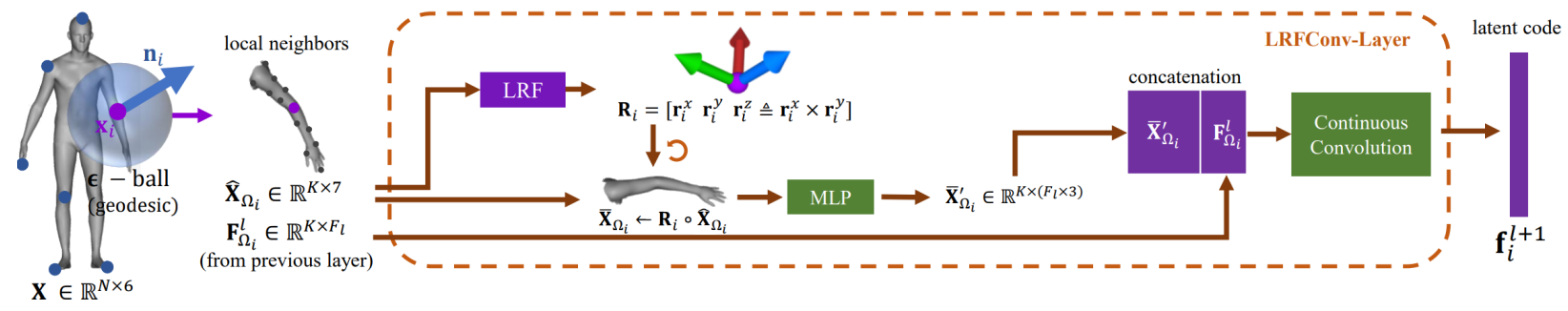

Figure 1: Our LRF-Conv layer. We treat a shape as a set of $3 \mathrm{D}$ coordinates and surface normals $\mathbf{X} \in \mathbb{R}^{N \times 6}$. Around each point $\mathbf{x}_{i} \in \mathbb{R}^{6} \equiv\left[x, y, z, n_{x}, n_{y}, n_{z}\right]^{\top}$ we consider a $3 \mathrm{D}$ geodesic local neighborhood $\Omega_{i}: \mathbf{X}_{\Omega_{i}}^{M}=\left\{\mathbf{x}_{k} \in \mathbf{X}: d\left(\mathbf{x}_{i}, \mathbf{x}_{k}\right)<\tau\right\}$, where $\tau$ is a threshold on the geodesic distance $d$. We then perform a farthest point sampling (FPS) on this set and retain $K$ neighbors. We denote this set $\mathbf{X}_{\Omega_{i}}=\operatorname{FPS}\left(\mathbf{X}_{\Omega_{i}}^{M}, K\right)$. We perform a de-mean operation, setting the coordinates of $\mathbf{x}_{i}$ as the local origin. We further augment each point with the geodesic distance to this new origin as well as the surface normal of the origin and let $\hat{\mathbf{x}}_{i j} \in \mathbb{R}^{7}=\left[\hat{x}_{i j}, \hat{y}_{i j}, \hat{z}_{i j}, \hat{n}_{i j}^{x}, \hat{n}_{i j}^{y}, \hat{n}_{i j}^{z}, g_{i j} \triangleq d\left(\mathbf{x}_{i}, \mathbf{x}_{i j}\right)\right]^{\top}$ represent the $j^{\text {th }}$ point in the local frame of $\mathbf{x}_{i}$. We use $\hat{\mathbf{X}}_{\Omega_{i}}=\left\{\hat{\mathbf{x}}_{i j}\right\}_{j \in \Omega_{i}}$ to refer to this augmented, centered local set and this is precisely the input to our LRF-Conv layer. Our layer takes as input a local reference frame (Tombari et al., 2010b) $\mathbf{R}_{i}$ assigned to the patch $i$. Next, we re-orient the input by $\mathbf{R}_{i}$ to get $\left\{\overline{\mathbf{x}}_{i j}\right\}_{j} \triangleq \overline{\mathbf{X}}_{\Omega_{i}}=\mathbf{R}_{i} \circ \hat{\mathbf{X}}_{\Omega_{i}}$. Note that the operator $\circ$ does not act on the distances $\left\{d_{i j}\right\}_{j}$ while rotating the rest. We then use an MLP per entity (coordinates, normals, distances) to extend the input description to match the latent dimension and concatenate these with features extracted in the previous layer $\mathbf{F}_{\Omega_{i}}^{l}$, where $\mathbf{F}_{\Omega_{i}}^{0} \triangleq \mathbf{0}$. This concatenated feature is the input to our trainable continuous convolution yielding the latent features (output) of this $(l+1)^{\text {th }}$ layer. We show in green the learnable modules whereas purple depicts the data containers.

\subsection{Continuous convolution}

Key to our approach is a continuous convolution operator. Realizing that the input points are merely samples from an underlying continuous surface, this makes a much more natural formulation than treating the points as an unstructured cloud. Several works have explored the use of learned continuous kernels. In Litany et al. (2017b); Digne et al. (2014) self similarities in the mesh patches were used via dictionary learning. More recently Masci et al. (2015); Boscaini et al. (2016a) studied the extraction of local geodesic patches for constructing the equivalent of a convolutional neural network for 2-dimensional manifolds. A generalized form of these was introduced in Monti et al. (2017). In Atzmon et al. (2018) a continuous processing of pointcloud was proposed by defining an extension operator that maps pointclouds to continuous volumetric functions. Another line of works parameterizes the continuous ambient function by another network. This concept was first introduced in Jia et al. (2016), where it was termed "dynamic filter as it allows to modify the convolution filters according to the input, instead of using fixed ones. In Dai et al. (2017) a deformation of the convolution kernel was used to dynamically react to the input image patch. A similar approach was taken for pointclouds in Thomas et al. (2019). Instead of dynamically modifying the kernel, Li et al. (2018) proposed a $\chi$-transformation in order to to canonicalize the input points. Our continuous convolution resembles the most this line of work, however differently from pointcloud based dynamic filters we utilize the mesh structure to enrich our point features as described in Section 3.

\section{LRFConv Layers}

At the core of our contribution lies a continuous convolution layer that operates on a locally rectified point set and its geodesics. We call this the LRFConv Layer and illustrate it in Figure 1. LRFConv receives a local patch $\hat{\mathbf{X}}_{\Omega_{i}}=\left\{\hat{\mathbf{x}}_{i j}\right\}_{j}=\left\{\hat{\mathbf{x}}_{i 1}, \hat{\mathbf{x}}_{i 2}, \ldots, \hat{\mathbf{x}}_{i K}\right\}$ that is already centered on a given reference point $\mathbf{x}_{i}$ as input. This local patch is composed of a collection of those $K$ points lying in the region $\Omega_{i}$ being subsampled using a farthest point sampling algorithm (Qi et al., 2017b; Moenning and Dodgson, 2003). Along with its $3 \mathrm{D}$ coordinates, each $j^{\text {th }}$ point in this patch also carries two additional pieces of geometric information: the surface normal $\hat{\mathbf{n}}_{i j} \in \mathbb{S}^{3}$ and the geodesic distance to the reference (center) point $d\left(\mathbf{x}_{i}, \mathbf{x}_{i j}\right)$, a quantity that is preserved under isometries. For a vertex $j$ centered around the $i^{\text {th }}$ vertex, this yields a 7 -dimensional point representation:

$$
\hat{\mathbf{x}}_{i j} \in \mathbb{R}^{7}=\left[\begin{array}{lllllll}
\hat{x}_{i j} & \hat{y}_{i j} & \hat{z}_{i j} & \hat{n}_{i j}^{x} & \hat{n}_{i j}^{y} & \hat{n}_{i j}^{z} & g_{i j} \triangleq d\left(\mathbf{x}_{i}, \mathbf{x}_{i j}\right)
\end{array}\right]^{\top}
$$


where subscript $i j$ refers to the index of the $j^{\text {th }}$ point in the neighborhood of $i^{\text {th }}$ vertex: $j \in \Omega_{i}$. In order to build resilience among six degrees of freedom (DoF) rotations we re-orient this patch using a local reference frame. To this end, we first compute an LRF for all points in the vertex set $\mathbf{X}$. Then each patch $\hat{\mathbf{X}}_{\Omega_{i}}$ is assigned an LRF in accordance with its index. The axes of this LRF are assembled into a rotation matrix $\mathbf{R}_{i} \in S O(3)$ which can be used to transform the patch to a canonical alignment: $\overline{\mathbf{X}}_{\Omega_{i}}=\mathbf{R}_{i} \circ \hat{\mathbf{X}}_{\Omega_{i}}$. Here the $\circ$ operator only acts on coordinates and normals separately. We then consider the aligned local patch $\overline{\mathbf{X}}_{\Omega_{i}} \triangleq\left\{\overline{\mathbf{x}}_{i j} \in \mathbb{R}^{7}\right\}_{j}$. Note that after such transformations $\overline{\mathbf{x}}_{i} \equiv \mathbf{o}$, a constant vector. We compute an intermediate feature representation for the whole patch using the entirety of the information collected up to this point and extend it with the help of three multi layer perceptrons (MLPs). We use one MLP per each of the coordinates, normals and geodesic distances in order to match the dimension of the latent features propagated from the previous layer, denoted as $\mathbf{F}_{\Omega_{i}}^{l} \in \mathbb{R}^{K \times F_{l}}$ where $F_{l}$ is the dimension of the features. This generates $\overline{\mathbf{X}}_{\Omega_{i}}^{\prime} \in \mathbb{R}^{K \times\left(F_{l} \times 3\right)}$. Note that an essential quantity in feeding forward the information generated in the previous layers to the later layers of our network is $\mathbf{F}_{\Omega_{i}}^{l}$. Thus, we concatenate the output of the said MLPs with $\mathbf{F}_{\Omega_{i}}^{l}$ and feed the resulting matrix into a continuous convolution operation producing the output features of this layer $\mathbf{f}_{i}^{l+1} \in \mathbb{R}^{F_{l+1}}$. Note that in the beginning we initialize the features to zeros: $\mathbf{F}_{\Omega_{i}}^{0} \triangleq \mathbf{0}$.

In the following, we will first present the details of our LRF computation and then dig deeper into the continuous convolutions.

Local Reference Frame (LRF) In order to introduce invariance to translations and rotations as well as building robustness to noise, many of the handcrafted descriptors rely on the estimation of a local coordinate system that varies equivariantly with the global transformation of the object. We use a similar idea to endow our deep features with invariances. A frame of reference (LRF) can be parameterized as a rotation matrix $\mathbf{R}_{i}=\left[\mathbf{r}_{i}^{x}, \mathbf{r}_{i}^{y}, \mathbf{r}_{i}^{z}\right] \in S O(3)$ where each column corresponds to an axis of the local coordinate frame. In our work, we switch between two LRFs depending on whether the data is real or synthetic. For scanned point sets, we use SHOT's LRF (Tombari et al., 2010b) thanks to its uniqueness and robustness to noise. The second kind that is suited to less noisy, synthetic meshes is inspired by Texturenet (Huang et al., 2019): The first axis is aligned with the surface normal at $\mathbf{x}_{i}: \mathbf{n}_{i}$. The second axis is determined by the direction of maximum curvature projected on the tangent plane defined by the surface normal (first axis). The third and the final axis is simply the cross product of the two: $\mathbf{r}_{i}^{z}=\mathbf{r}_{i}^{x} \times \mathbf{r}_{i}^{y}$. Such LRF construction reduces the degree of ambiguity from four as in (Huang et al., 2019) to two.

Continuous Graph Convolution An important portion of the success of the CNNs is attributed to the $2 \mathrm{D}$ convolutions that are well suited to the structured grid underlying the pixels. Unfortunately, for unstructured 3D data, defining such a grid is not trivial and hence the primary tools for point set processing such as PointNet (Qi et al., 2017a) prefer to ignore the domain and apply point-wise convolutions. Yet, taking into account the neighborhood structure is shown to be advantageous (Qi et al., 2017b). Thanks to the availability of the mesh structure, we could define an unstructured convolution analogous to the $2 \mathrm{D}$ that considers the mesh surface. To this end, we use continuous graph convolutions., whose details we show in Figure 2 and explain below.

As discussed in the introduction of this section, we first extract a patch $\mathbf{X}_{\Omega_{i}}^{M}=\left\{\mathbf{x}_{k} \in \mathbf{X}: d\left(\mathbf{x}_{i}, \mathbf{x}_{k}\right)<\tau\right\}$ according to the geodesic distance $\tau$ of the reference point $\mathbf{x}_{i}$. The we use FPS to get $\mathrm{K}$ points within $\mathbf{X}_{\Omega_{i}}^{M}$ and center them given $\mathbf{x}_{i}$ to get $\hat{\mathbf{X}}_{\Omega_{i}}$. After that, we rotate $\hat{\mathbf{X}}_{\Omega_{i}}$ using the LRF to obtain $\overline{\mathbf{X}}_{\Omega_{i}}$. $\overline{\mathbf{X}}_{\Omega_{i}}$ carries four feature components per each point in the neighborhood of $\mathbf{x}_{i}$ : the coordinate $\mathbf{v}_{i j}$, the aligned normal $\overline{\mathbf{n}}_{i j}$, the geodesic distance $g_{i j}$, and the feature from previous layer $\mathbf{f}_{i j}^{l}$. They could be expressed as $\mathbf{v}_{i j}=\bar{x}_{i j}-\bar{x}_{i}, \overline{\mathbf{n}}_{i j}=\mathbf{R}_{i} \hat{\mathbf{n}}_{i j}$, and $g_{i j}=d\left(\overline{\mathbf{x}}_{i}, \overline{\mathbf{x}}_{i j}\right)$. Note that $\mathbf{f}_{i j}^{l}$ is a rotation invariant feature. We will justify choosing these four feature components in Section 5.5. In our implementation, the first layer omits this feature. Though, signals such as color could be whenever available. For the following layers, $\mathbf{f}_{i j}^{l}(l>0)$ is the computed rotation invariant feature. If $\mathbf{f}_{i j}^{l}$ exists, then we first use three MLPs $\left(M L P_{v b}, M L P_{n b}\right.$, and $\left.M L P_{g}\right)$ to expand the dimension of $\mathbf{v}_{i j}, \overline{\mathbf{n}}_{i j}$, and $g_{i j}$ to match the dimension of $\mathbf{f}_{i j}^{l}$. If $\mathbf{f}_{i j}^{l}$ does not exist, we expand the dimension of $\mathbf{v}_{i j}$ to 9 . This can help making the network treat each feature's components equally in the next step. After concatenating the expanded input descriptions $\overline{\mathbf{x}}_{i j}^{\prime}$ and $\mathbf{f}_{i j}^{l}$, we use another $M L P_{w}$ to regress a weight matrix whose size is is $F_{l} \times F_{l+1}$. Subsequently, we apply a discrete convolution 
operation between this convolution weights matrix and the input features:

$$
\mathbf{f}_{i}^{l+1}=\sum_{j=1}^{K} M L P_{w}\left(\left[\overline{\mathbf{x}}_{i j}^{\prime}, \mathbf{f}_{i j}^{l}\right]\right)\left[\overline{\mathbf{x}}_{i j}^{\prime}, \mathbf{f}_{i j}^{l}\right]
$$

With that we update the feature at the reference points $\mathbf{x}_{i}$. The whole process is depicted in Figure 2 .

\section{Network Architecture}

Our network architecture as shown in Figure 3 consumes each local part separately and involves stacking of the LRF-Conv layers with skip connections. The upper skip links marked in brown denote that the coordinates, normals, and geodesic distances are also fed forward. In addition, by speaking of LRFConvBN in Figure 3, it means a LRFConv layer followed by a batch normalization layer Ioffe and Szegedy (2015) ,and a non-linear layer, in our case ReLU Nair and Hinton (2010). The part of network before branching into task specific modules is what we call the learned shape descriptor (LSD). The architecture of LSD is motivated by ResNet (He et al., 2016). As the layers deepen, we gradually increase the perception field of the network and the length of the feature while having skip connections to increase the depth of the network. This avoids the vanishing gradient problem. Once the LSD is extracted for each vertex (local patch) on the shape, we use it for two tasks, human body segmentation and shape correspondence.

Part Segmentation The fully connected network for part segmentation is composed of 7 residual blocks. For each block the dimension of the output feature is reduced by half with the first dimension being 512 . In the last residual block, the dimension equals to the number of classes $M$ which is 8 . And a softmax layer is followed. We get an output label $\mathbf{s}_{i}$ for each patch anchored at $\mathbf{x}_{i}$. For the segmentation task, we minimize the cross-entropy loss between the output predictions $\left\{s_{i}\right\}$ and the ground truth segmentation labels $\left\{y_{i}\right\}$.

$$
\ell=-\frac{1}{M} \sum_{i=1}^{M} y_{i} \log s_{i}
$$

Correspondence Estimation The fully connected network for estimating correspondence consists of 7 residual blocks. In order to have a fair comparison with FMNet (Litany et al., 2017a), the dimension of the output / feature of each block is set to 352. This is identical to the length of the latent feature used in FMNet. We use the shared weights of LSD followed by the fully connected network to extract the feature from the target shape $\mathcal{Y}$ and the source shape $\mathcal{X}$. Then we follow the loss function proposed in FMNet:

$$
\ell=\frac{1}{|\mathcal{X}|}\left\|\mathbf{P} \circ\left(\mathbf{D}_{\mathcal{Y}} \Pi^{*}\right)\right\|_{F}^{2}
$$

Note that our correspondence estimation approach resembles FMNet's. However, in addition to our continuous convolutions, we avoid using the handcrafted SHOT descriptors and replace them with LSDs.

\section{Experimental Evaluation}

\subsection{Datasets}

We demonstrate the efficacy of our learned descriptor on two cornerstone tasks in shape analysis: dense shape correspondence and part segmentation. To this end, we utilize two datasets.

Part Segmentation. For the segmentation task, we use the human segmentation benchmark introduced in Maron et al. (2017). This dataset consists of 370 models fused from multiple human shape collections: SCAPE (Anguelov et al., 2005), FAUST (Bogo et al., 2014), MIT animation datasets (Vlasic et al., 2008) and Adobe Fuse (Adobe, 2016). All models were manually segmented into eight parts as prescribed by Kalogerakis et al. (2010). The test set is the 18 human models from the SHREC dataset (Giorgi et al.). The variety of data sources makes the problem especially challenging as each collection has a different sampling and appearance. Moreover, the SHREC dataset was used solely for testing which calls for a high generalization ability. 


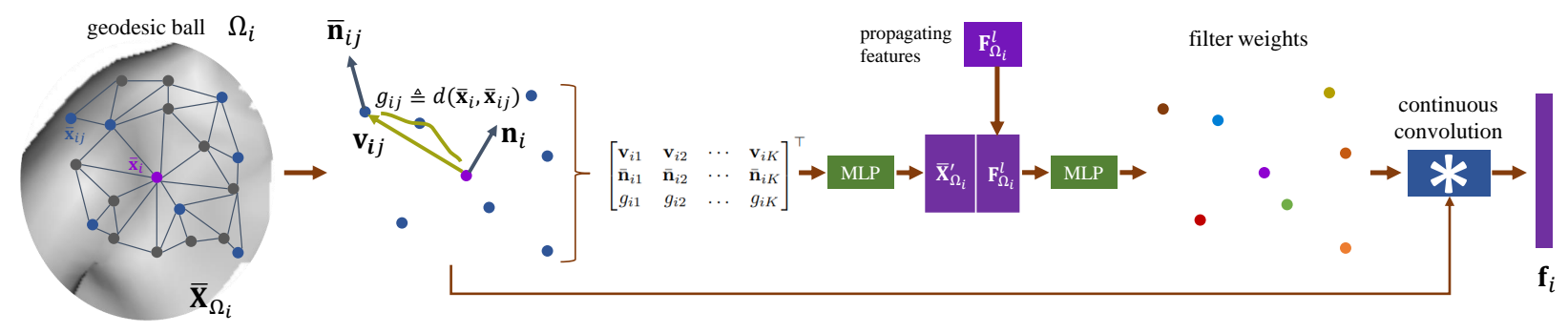

Figure 2: Details of our continuous geodesic convolution. The figure shows in more detail the computation of the input to the MLP and subsequently to the convolution in the unstructured domain. Our MLP that regresses the convolution kernel learns a mapping from the high dimensional point/patch representation to a matrix of weights.

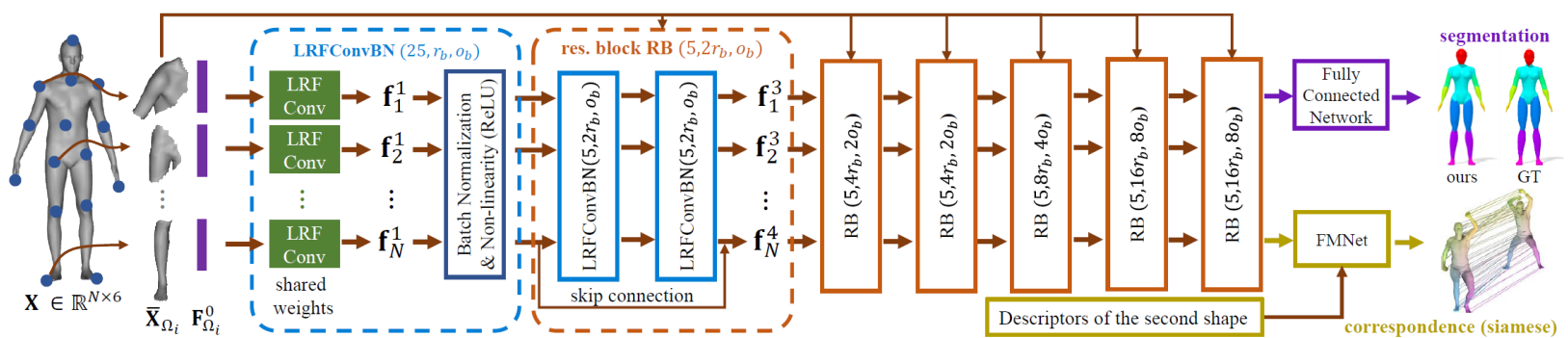

Figure 3: Entirety of our architecture. We extract a local patch centered around each point by querying a geodesic neighborhood. These patches are sent into a sequence of LRFConv layers followed by a batch normalization (BN) and ReLU non-linearity. We also add skip connections to be able to increase the depth and avoid the vanishing gradients. After 13 layers of LRFConv we arrive at our latent features which can be used to address common tasks such as human body segmentation or correspondence estimation. When LRFConv is followed by a BN and ReLU, we call this an LRFConvBN layer and parametrize it by three respective arguments: $K$ the number of points in the patch, $r_{b}=0.003$ the base radius that determines the size of the neighborhood, $o_{b}=32$ where $o_{l}=\lambda o_{b}$ sets the dimension of the output features. The residual blocks (RB) that are composed by two LRFConBNs are similarly parametrized. Note that for correspondence estimation we have a weight sharing siamese architecture where the latent features of a paired shape are fed into a deep functional map network (Litany et al., 2017a) along with the features of the base (current) mesh.

Shape Correspondence. To showcase our descriptor learning module in the task of shape matching we use the FAUST dataset (Bogo et al., 2014). The data contains 100 human high resolution scans belonging to 10 different individuals at 10 different poses each. The scans were all registered to a parametric model with 6890 vertices and consistent triangulation. We call this set "Synthetic FAUST". We also test our method on the more challenging set of the original scans.

\subsection{Part Segmentation}

Given an input mesh we use our network to predict for each vertex the part segment it belongs to. At train time, we select 2000 random points from each mesh as input to the network, and use the corresponding segmentation label as the supervision signal. We train our network for 200 epochs. In all our experiments we use the Adam optimizer (Kingma and $\mathrm{Ba}, 2014$ ) with a fixed learning rate of $10^{-3}, \beta_{1}=0.9, \beta_{2}=0.999$, and $\epsilon=10^{-8}$. We compare our results with the two variations of MDGCNN (Poulenard and Ovsjanikov, 2019) as proposed by the authors, using either raw 3D coordinates or precomputed SHOT (Tombari et al., $2010 \mathrm{~b})$ descriptors. To better understand the influence of the continuous convolution module (CC) we also compare with a simplified version of our pipeline, where the continuous convolution is replaced by a standard PointNet (PN) (Qi et al., 2017a). Our results are summarized in Table 1. It can be seen that our method in both of its forms outperforms MDGCNN, while using the continuous convolution further boosts the performance.

Importantly, we achieve this by using raw 3D coordinates as input and by which bridges the gap reported in MDGCNN allowing to remove the dependence on manually designed features. This desired behaviour 


\begin{tabular}{l|c|c} 
Method & Input feature & Accuracy \\
\hline MDGCNN (Poulenard and Ovsjanikov, 2019) & 3D coords & 88.61 \\
MDGCNN (Poulenard and Ovsjanikov, 2019) & SHOT $_{12}$ & 89.47 \\
\hline Ours PN & 3D coords & 89.69 \\
Ours CC & 3D coords & $\mathbf{8 9 . 8 8}$
\end{tabular}

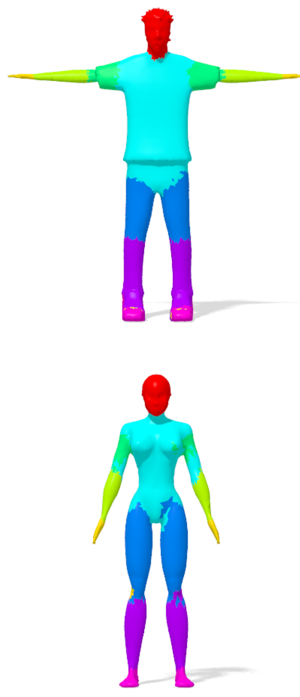

PointNet++
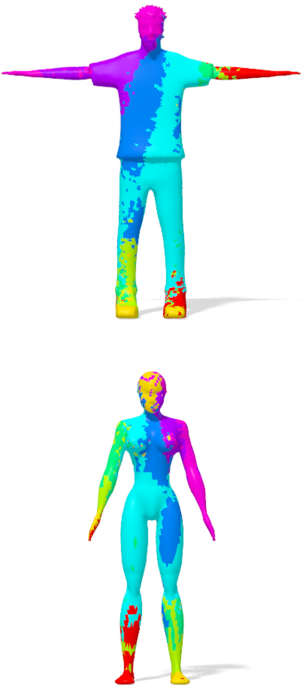

DGCNN
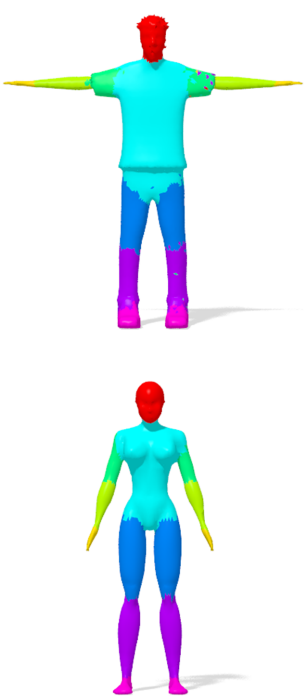

Ours (PN)

Table 1: We compare our results with the two variations of MDGCNN (Poulenard and Ovsjanikov, 2019) as proposed by the authors, using either raw $3 \mathrm{D}$ coordinates or precomputed SHOT (Tombari et al., 2010b) descriptors. To better understand the influence of the continuous convolution module (CC) we also compare with a simplified version of our pipeline, where the continuous convolution is replaced by a standard PointNet (PN) (Qi et al., 2017a).

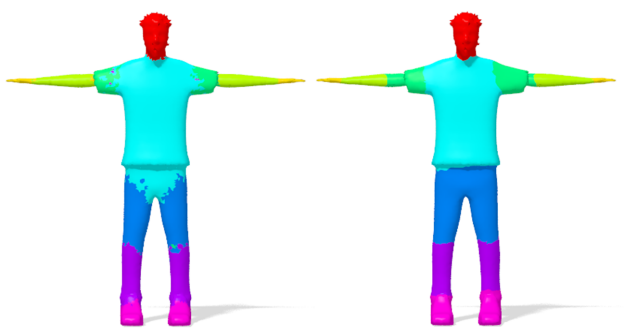

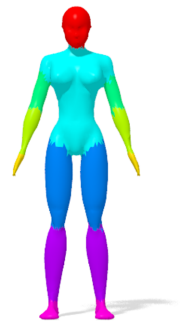

Ours (CN)

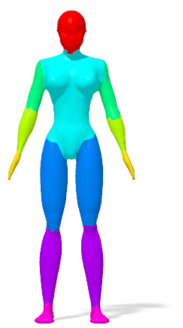

Groundthruth

Figure 4: A qualitative evaluation of our method on human body segmentation comparing to other learning-based approaches.

is expected since our network imitates the design philosophy of SHOT. We further present a qualitative evaluation of the part segmentation in Figure 4.

\subsection{Rotation invariance}

As described in the introduction, depending on the dataset and task at hand, a descriptor should be invariance to different transformations. In the case of human body models and part segmentation it is natural to ask for invariance to rigid transformations and articulations. While the latter is achieved through learning from examples, the former is taken care of by construction using our proposed LRF. Baking invariance into the descriptor by construction is not only natural to the problem setting but also more sample efficient. To see this, we take two baseline methods which are not rotation invariant, and train them with and without rotation augmentation. We also create an augmented test set, where each shape has been rotated in 128 different angles. We summarize the results of these methods and ours in Table 2. As can be seen, when the non rotation invariant networks are tested on rotated examples their performance drops significantly. Adding rotation at train time helps shrinking the performance gap however, both still under perform compared to our rotation invariant solution.

Looking closely at the results of PointNet++ (Qi et al., 2017b) one notices that the results improve on the non-rotated test set by adding the augmentation at train time. This is explained by the fact that the models in both train and test sets are either upright or lying down but with very different distribution between the two poses. What is less intuitive is why this improvement is not achieved for DGCNN. From our experiments we conclude that the method could not benefit from the augmentation and instead reduced 


\begin{tabular}{c|c|c|c|c|c} 
Train with rotation & Test with rotation & PointNet++ & DGCNN & Ours $(\mathrm{PN})$ & Ours $(\mathrm{CC})$ \\
\hline Yes & Yes & 85.35 & 43.88 & & \\
Yes & No & 85.85 & 36.99 & & \\
No & Yes & 56.95 & 36.65 & 89.69 & $\mathbf{8 9 . 8 8}$ \\
No & No & 75.81 & 66.35 & &
\end{tabular}

Table 2: The accuracy on human body segmentation of different learning-based approaches under training and test with and without rotation augmentation

to solving the average case, perhaps due to limited capacity. In Figure 5 we compare the results of our network with DGCNN on each of the 18 test shapes. To better visualize the effect of the rotation angle we sort the test samples (x-axis) according to the performance of DGCNN. This clearly shows a gap between samples where the input shape was lying down (first 6 examples) and the ones which were upright.

\subsection{Shape matching}

Finding dense shape correspondences between a pair of shapes is one of the most important and most explored problems in shape analysis. As described earlier, state of the art methods utilize hand crafted descriptors as input. Here, instead, we propose to learn the descriptor directly from raw 3D coordinates by utilizing our proposed LRFConv. Our descriptor can in principle be combined with any matching pipeline. In this work we make use of FMNet (Litany et al., 2017a), one of the best performing methods in the task of shape matching. Specifically, it accepts a pair of shapes as input together with their computed Laplacian eigenfunctions and per-point features. Then, both shape features are passed through a (siamese) feed forward network to get refined descriptors. These, in turn, are used to compute a functional map aligning the shape eignefunctions. Finally, these are used to predict a point-to-point soft-correspondences which are converted to matchies by taking the maximal probability per point. In the original work of (Litany et al., 2017a) and its unsupervised follow up (Halimi et al., 2018) SHOT descriptors were used. Here, instead, we replace it with our learned descriptors and train the network in an end-to-end fashion.

Synthetic FAUST We first demonstrate our performance on the synthetic FAUST dataset described in 5.1. We follow the evaluation protocol as prescribed by Monti et al. (2017) were the 100 models are split into 80 train and 20 test shapes, and the matching is performed with respect to a single fixed null shape. We train the network for 200 epochs using the same optimization hyper parameters as described in 5.2. The results are summarized in Figure 6. As can be seen, by using our proposed descriptor we were able to improve upon the results of FMNet with SHOT. We include the performance of other methods for the sake of completeness.

In Figure 7 we show a qualitative evaluation of our matching results.

\subsection{Ablation Study}

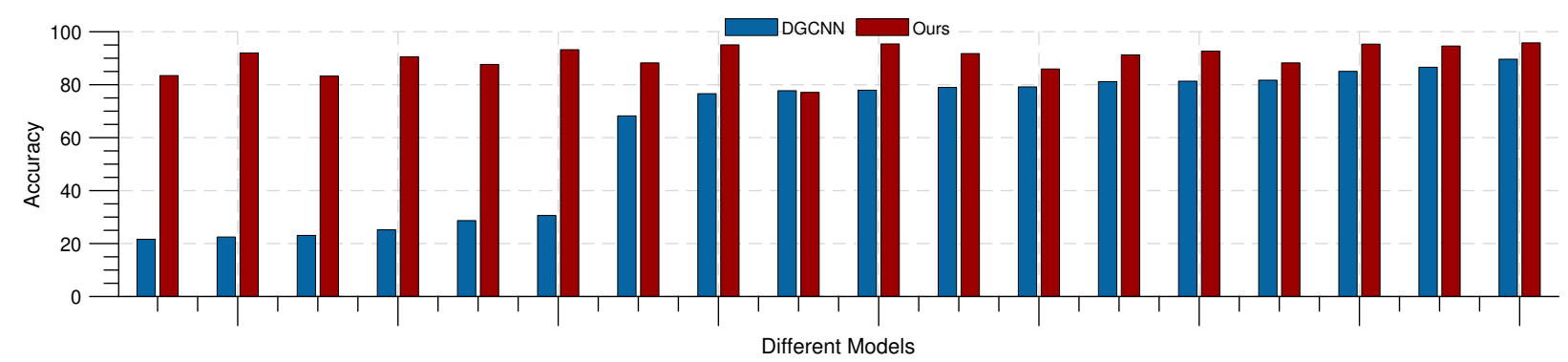

Figure 5: We compare the results of our network with DGCNN on each of the 18 test shapes. To better visualize the effect of the rotation angle we sort the test samples (x-axis) according to the performance of DGCNN. This clearly shows a gap between samples where the input shape was lying down (first 6 examples) and the ones which were upright. 


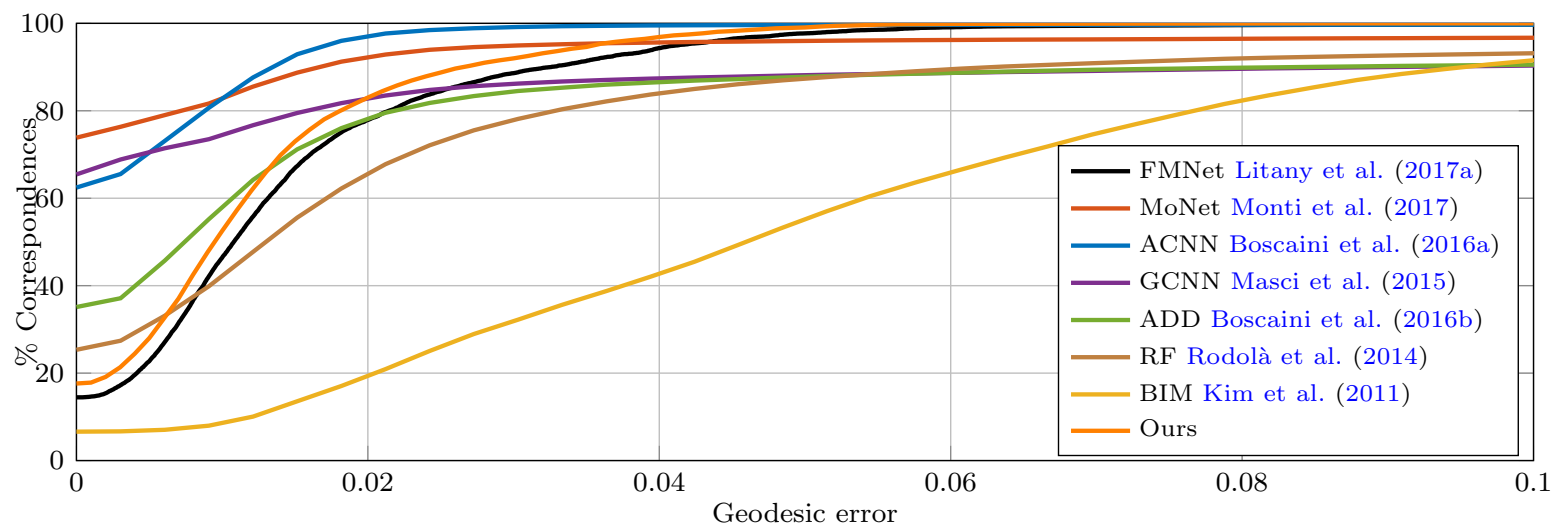

Figure 6: Comparison with learning-based approaches on the FAUST humans dataset. By using our proposed descriptor, we were able to improve upon the results of FMNet with SHOT.
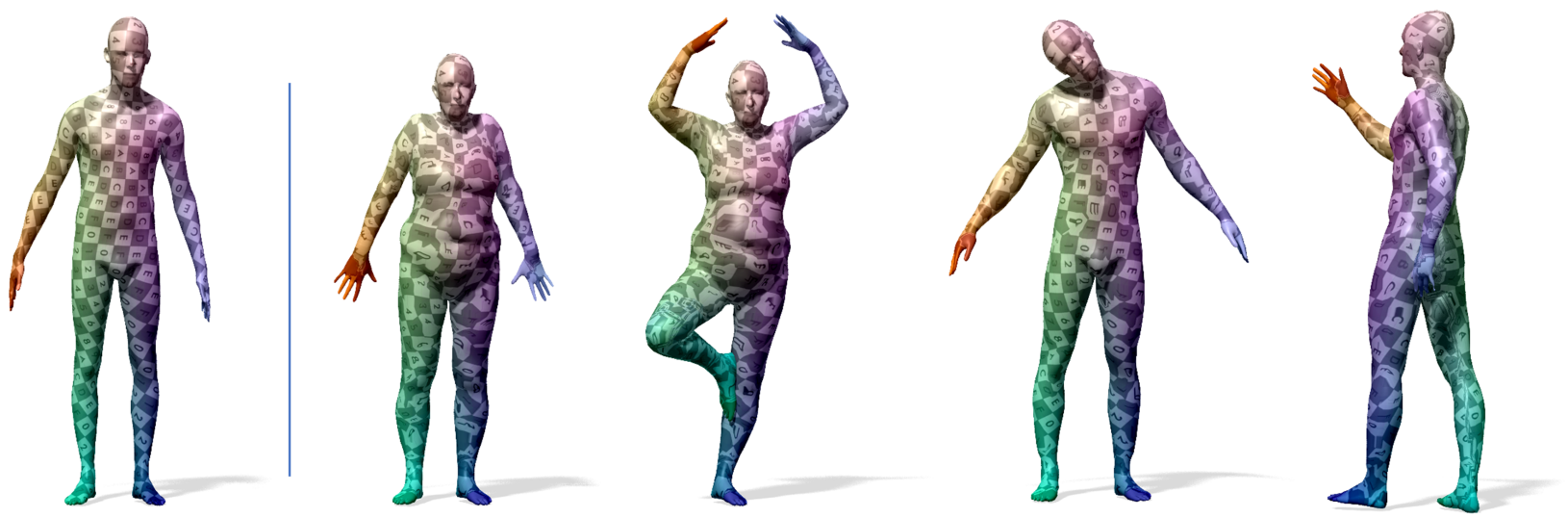

Figure 7: A qualitative evaluation of our matching results on synthetic FAUST. On the left of the vertical border is a textured target shape. To the right are four source shapes from the test set with their texture pulled back from the target according to the recovered correspondences.

In this section we provide analysis of the design choices made when constructing our descriptor learning network. To this end, we utilize the synthetic FAUST dataset and the task of part segmentation. Since we are working in a simplified setting with only one dataset, we make two modifications to the network. First, we reduce the base feature dimensionality (see $o_{b}$ in Figure 4) from 32 to 4. Second, since the number of vertices is kept fixed we use all vertices instead of subsampling a $2 \mathrm{~K}$ subset. To evaluate the importance of each ingredient, we retrain and evaluate the network performance with and without it. Results are summarized in Table 3. It can be seen that the accuracy achieved with both $\mathrm{CC}$ and PN, is very high.

The importance of the rest of the components in descending order is: the coordinates $\mathbf{v}_{i j}$, removing feature propagation from previous layers $\mathbf{f}_{i j}^{l}$, LRF, normals $\mathbf{n}_{i j}$, and geodesic distance to the central vertex $g_{i j}$. As expected, the coordinate $\mathbf{v}_{i j}$ is playing the biggest role as it is holds the most information of the local patch geometry. Another motivation to include normals comes from the results reported in Koch et al. (2019), where it was shown that all state-of-the-art networks struggle with accurately estimating the normal of a patch. The results justify the inclusion of each of the components. 


\section{Conclusion}

In this work we have studied the problem of learning shape descriptors. A main motivation for this work was the fact that sate-of-the-art learning based techniques were still relying on hand crafted descriptors. Here, we showed that this is mainly due to the usage of the LRF. By baking the computation of an LRF into the design of the network we were able to bridge the gap and outperform manual descriptor- based methods with using raw mesh features: coordinates, normals, and geodesic distances. In addition, we introduced a continuous convolution kernel which allows the filters to dynamically react to the input features. We demonstrated the performance of our proposed method on two important tasks: shape matching and part segmentation. Albeit the usage of a continuous convolution, current method including ours, still rely heavily on the set of sampling points and the sampling method (FPS in our case). This is of course an unwanted behaviour as the result should depend on the underlying surface. In future work we plan to explore this direction.

\section{References}

Adobe, 2016. Adobe fuse 3d characters. URL: https://www.mixamo.com/\#/.

Anguelov, D., Srinivasan, P., Koller, D., Thrun, S., Rodgers, J., Davis, J., 2005. Scape: shape completion and animation of people, in: ACM transactions on graphics (TOG), ACM. pp. 408-416.

Atzmon, M., Maron, H., Lipman, Y., 2018. Point convolutional neural networks by extension operators. arXiv preprint arXiv:1803.10091

Aubry, M., Schlickewei, U., Cremers, D., 2011. The wave kernel signature: A quantum mechanical approach to shape analysis, in: 2011 IEEE international conference on computer vision workshops (ICCV workshops), IEEE. pp. $1626-1633$.

Bogo, F., Romero, J., Loper, M., Black, M.J., 2014. Faust: Dataset and evaluation for 3d mesh registration, in: Proceedings of the IEEE Conference on Computer Vision and Pattern Recognition, pp. 3794-3801.

Boscaini, D., Masci, J., Rodolà, E., Bronstein, M., 2016a. Learning shape correspondence with anisotropic convolutional neural networks, in: Advances in Neural Information Processing Systems, pp. 3189-3197.

Boscaini, D., Masci, J., Rodolà, E., Bronstein, M.M., Cremers, D., 2016b. Anisotropic diffusion descriptors, in: Computer Graphics Forum, Wiley Online Library. pp. 431-441.

Bronstein, A.M., Bronstein, M.M., Guibas, L.J., Ovsjanikov, M., 2011. Shape google: Geometric words and expressions for invariant shape retrieval. ACM Transactions on Graphics (TOG) 30, 1.

Bronstein, M.M., Bruna, J., LeCun, Y., Szlam, A., Vandergheynst, P., 2017. Geometric deep learning: going beyond euclidean data. IEEE Signal Processing Magazine 34, 18-42.

Bronstein, M.M., Kokkinos, I., 2010. Scale-invariant heat kernel signatures for non-rigid shape recognition, in: 2010 IEEE Computer Society Conference on Computer Vision and Pattern Recognition, IEEE. pp. 1704-1711.

Bruna, J., Zaremba, W., Szlam, A., LeCun, Y., 2013. Spectral networks and locally connected networks on graphs. arXiv preprint arXiv:1312.6203 .

Choy, C., Park, J., Koltun, V., 2019. Fully convolutional geometric features, in: The IEEE International Conference on Computer Vision (ICCV), pp. 8958-8966.

Dai, J., Qi, H., Xiong, Y., Li, Y., Zhang, G., Hu, H., Wei, Y., 2017. Deformable convolutional networks, in: Proceedings of the IEEE international conference on computer vision, pp. 764-773.

Defferrard, M., Bresson, X., Vandergheynst, P., 2016. Convolutional neural networks on graphs with fast localized spectral filtering, in: Advances in neural information processing systems, pp. 3844-3852.

Deng, H., Birdal, T., Ilic, S., 2018a. Ppf-foldnet: Unsupervised learning of rotation invariant 3d local descriptors, in: European conference on computer vision (ECCV).

Deng, H., Birdal, T., Ilic, S., 2018b. Ppfnet: Global context aware local features for robust 3d point matching, in: IEEE Conference on Computer Vision and Pattern Recognition (CVPR), pp. 195-205.

Deng, H., Birdal, T., Ilic, S., 2019. 3d local features for direct pairwise registration, in: IEEE Conference on Computer Vision and Pattern Recognition (CVPR).

Digne, J., Chaine, R., Valette, S., 2014. Self-similarity for accurate compression of point sampled surfaces, in: Computer Graphics Forum, Wiley Online Library. pp. 155-164.

Giorgi, D., Biasotti, S., Paraboschi, L., . Shape retrieval contest 2007: Watertight models track .

Gojcic, Z., Zhou, C., Wegner, J.D., Wieser, A., 2019. The perfect match: 3d point cloud matching with smoothed densities, in: IEEE Conference on Computer Vision and Pattern Recognition (CVPR), pp. 5545-5554.

Guo, Y., Sohel, F.A., Bennamoun, M., Wan, J., Lu, M., 2013. Rops: A local feature descriptor for 3d rigid objects based on rotational projection statistics, in: Communications, Signal Processing, and their Applications (ICCSPA), 2013 1st International Conference on, IEEE. pp. 1-6.

Halimi, O., Litany, O., Rodol'a, E., Bronstein, A., Kimmel, R., 2018. Self-supervised learning of dense shape correspondence. arXiv preprint arXiv:1812.02415

Hanocka, R., Hertz, A., Fish, N., Giryes, R., Fleishman, S., Cohen-Or, D., 2019. Meshcnn: A network with an edge. ACM Transactions on Graphics (TOG) 38, 90. 
He, K., Zhang, X., Ren, S., Sun, J., 2016. Deep residual learning for image recognition, in: Proceedings of the IEEE conference on computer vision and pattern recognition, pp. 770-778.

Henaff, M., Bruna, J., LeCun, Y., 2015. Deep convolutional networks on graph-structured data. arXiv preprint arXiv:1506.05163

Huang, C.H., Tombari, F., Navab, N., 2015. Repeatable local coordinate frames for 3d human motion tracking: From rigid to non-rigid, in: 2015 International Conference on 3D Vision, IEEE. pp. 371-379.

Huang, J., Zhang, H., Yi, L., Funkhouser, T., Nießner, M., Guibas, L.J., 2019. Texturenet: Consistent local parametrizations for learning from high-resolution signals on meshes, in: Proceedings of the IEEE Conference on Computer Vision and Pattern Recognition, pp. 4440-4449.

Ioffe, S., Szegedy, C., 2015. Batch normalization: Accelerating deep network training by reducing internal covariate shift. CoRR abs/1502.03167. URL: http://arxiv.org/abs/1502.03167, arXiv:1502.03167.

Jia, X., De Brabandere, B., Tuytelaars, T., Gool, L.V., 2016. Dynamic filter networks, in: Advances in Neural Information Processing Systems, pp. 667-675.

Johnson, A.E., Hebert, M., 1999. Using spin images for efficient object recognition in cluttered 3d scenes. IEEE Transactions on pattern analysis and machine intelligence 21, 433-449.

Kalogerakis, E., Hertzmann, A., Singh, K., 2010. Learning 3d mesh segmentation and labeling, in: ACM Transactions on Graphics (TOG), ACM. p. 102.

Khoury, M., Zhou, Q.Y., Koltun, V., 2017. Learning compact geometric features, in: IEEE International Conference on Computer Vision (ICCV).

Kim, V.G., Lipman, Y., Funkhouser, T., 2011. Blended intrinsic maps, in: ACM Transactions on Graphics (TOG), ACM. p. 79.

Kingma, D.P., Ba, J., 2014. Adam: A method for stochastic optimization. arXiv preprint arXiv:1412.6980 .

Koch, S., Matveev, A., Jiang, Z., Williams, F., Artemov, A., Burnaev, E., Alexa, M., Zorin, D., Panozzo, D., 2019. Abc: A big cad model dataset for geometric deep learning, in: Proceedings of the IEEE Conference on Computer Vision and Pattern Recognition, pp. 9601-9611.

Li, Y., Bu, R., Sun, M., Chen, B., 2018. Pointcnn. arXiv preprint arXiv:1801.07791 .

Litany, O., Remez, T., Rodola, E., Bronstein, A., Bronstein, M., 2017a. Deep functional maps: Structured prediction for dense shape correspondence, in: Proceedings of the IEEE International Conference on Computer Vision, pp. 5659-5667.

Litany, O., Remez, T., Tal, Bronstein, A., 2017b. Cloud dictionary: Sparse coding and modeling for point clouds. SPARS .

Litman, R., Bronstein, A.M., 2013. Learning spectral descriptors for deformable shape correspondence. IEEE transactions on pattern analysis and machine intelligence 36, 171-180.

Maron, H., Galun, M., Aigerman, N., Trope, M., dym, N., , Yumer, E., Kim, V., Lipman, Y., 2017. Convolutional neural networks on surfaces via seamless toric covers. ACM Transactions on Graphics (Proceedings of SIGGRAPH 2017) .

Masci, J., Boscaini, D., Bronstein, M., Vandergheynst, P., 2015. Geodesic convolutional neural networks on riemannian manifolds, in: Proceedings of the IEEE international conference on computer vision workshops, pp. 37-45.

Melzi, S., Spezialetti, R., Tombari, F., Bronstein, M.M., Stefano, L.D., Rodola, E., 2019. Gframes: Gradient-based local reference frame for $3 \mathrm{~d}$ shape matching, in: The IEEE Conference on Computer Vision and Pattern Recognition (CVPR).

Mian, A., Bennamoun, M., Owens, R., 2010. On the repeatability and quality of keypoints for local feature-based 3d object retrieval from cluttered scenes. International Journal of Computer Vision 89, 348-361.

Moenning, C., Dodgson, N.A., 2003. Fast marching farthest point sampling. Technical Report. University of Cambridge, Computer Laboratory.

Monti, F., Boscaini, D., Masci, J., Rodola, E., Svoboda, J., Bronstein, M.M., 2017. Geometric deep learning on graphs and manifolds using mixture model cnns, in: Proceedings of the IEEE Conference on Computer Vision and Pattern Recognition, pp. $5115-5124$.

Nair, V., Hinton, G.E., 2010. Rectified linear units improve restricted boltzmann machines, in: Proceedings of the 27th international conference on machine learning (ICML-10), pp. 807-814.

Petrelli, A., Di Stefano, L., 2011. On the repeatability of the local reference frame for partial shape matching, in: 2011 International Conference on Computer Vision, IEEE. pp. 2244-2251.

Poulenard, A., Ovsjanikov, M., 2019. Multi-directional geodesic neural networks via equivariant convolution. ACM Transactions on Graphics (TOG) 37, 236 .

Qi, C.R., Su, H., Mo, K., Guibas, L.J., 2017a. Pointnet: Deep learning on point sets for 3d classification and segmentation, in: Proceedings of the IEEE Conference on Computer Vision and Pattern Recognition, pp. 652-660.

Qi, C.R., Yi, L., Su, H., Guibas, L.J., 2017b. Pointnet++: Deep hierarchical feature learning on point sets in a metric space, in: Advances in neural information processing systems, pp. 5099-5108.

Rodolà, E., Rota Bulo, S., Windheuser, T., Vestner, M., Cremers, D., 2014. Dense non-rigid shape correspondence using random forests, in: Proceedings of the IEEE Conference on Computer Vision and Pattern Recognition, pp. 4177-4184.

Rustamov, R.M., 2007. Laplace-beltrami eigenfunctions for deformation invariant shape representation, in: Proceedings of the fifth Eurographics symposium on Geometry processing, Eurographics Association. pp. 225-233.

Rusu, R.B., Blodow, N., Beetz, M., 2009. Fast point feature histograms (fpfh) for 3d registration, in: Robotics and Automation, 2009. ICRA'09. IEEE International Conference on, IEEE. pp. 3212-3217.

Rusu, R.B., Marton, Z.C., Blodow, N., Beetz, M., 2008. Persistent Point Feature Histograms for 3D Point Clouds, in: Proceedings of the 10th International Conference on Intelligent Autonomous Systems (IAS-10), Baden-Baden, Germany

Sun, J., Ovsjanikov, M., Guibas, L., 2009. A concise and provably informative multi-scale signature based on heat diffusion, in: Computer graphics forum, Wiley Online Library. pp. 1383-1392.

Thomas, H., Qi, C.R., Deschaud, J.E., Marcotegui, B., Goulette, F., Guibas, L.J., 2019. Kpconv: Flexible and deformable 
convolution for point clouds. arXiv preprint arXiv:1904.08889 .

Tombari, F., Salti, S., Di Stefano, L., 2010a. Unique shape context for 3d data description, in: Proceedings of the ACM workshop on 3D object retrieval, ACM. pp. 57-62.

Tombari, F., Salti, S., Di Stefano, L., 2010b. Unique signatures of histograms for local surface description, in: European conference on computer vision, Springer. pp. 356-369.

Verma, N., Boyer, E., Verbeek, J., 2018. Feastnet: Feature-steered graph convolutions for 3d shape analysis, in: Proceedings of the IEEE Conference on Computer Vision and Pattern Recognition, pp. 2598-2606.

Vlasic, D., Baran, I., Matusik, W., Popović, J., 2008. Articulated mesh animation from multi-view silhouettes, in: ACM Transactions on Graphics (TOG), ACM. p. 97.

Wang, S., Suo, S., Ma, W.C., Pokrovsky, A., Urtasun, R., 2018. Deep parametric continuous convolutional neural networks, in: Proceedings of the IEEE Conference on Computer Vision and Pattern Recognition, pp. 2589-2597.

Zeng, A., Song, S., Nießner, M., Fisher, M., Xiao, J., Funkhouser, T., 2017. 3DMatch: Learning Local Geometric Descriptors from RGB-D Reconstructions, in: IEEE Conference on Computer Vision and Pattern Recognition (CVPR).

Zhao, Y., Birdal, T., Lenssen, J.E., Menegatti, E., Guibas, L., Tombari, F., 2019. Quaternion equivariant capsule networks for 3d point clouds. arXiv preprint arXiv:1912.12098 . 